\title{
Alteration of the phenolic fraction of extra virgin olive oil subjected to frying conditions
}

Inmaculada Criado-Navarro ${ }^{1,2,3,4}$, María Asunción López-Bascón ${ }^{1,2,3,4}$, Carlos Augusto Ledesma-Escobar ${ }^{1,2,3,4}$, Feliciano Priego-Capote ${ }^{* 1,2,3,4}$

${ }^{1}$ Department of Analytical Chemistry, University of Córdoba, Córdoba E-14071, Spain

${ }^{2}$ Nanochemistry University Institute (IUNAN), University of Córdoba, Córdoba E-14071, Spain

${ }^{3}$ Maimónides Institute of Biomedical Research (IMIBIC), Reina Sofía University Hospital, University of Córdoba, Córdoba E-14071, Spain

${ }^{4}$ CIBER of Frailty and Healthy Ageing (CIBERFES), Carlos III Health Institute, Madrid E28029, Spain

*Corresponding author: F. Priego-Capote (feliciano.priego@uco.es).

Table S1. LC-MS/MS parameters for determination of phenolic compounds in olive oil.

\begin{tabular}{lccccc}
\hline \multicolumn{1}{c}{ Compound } & $\begin{array}{c}\text { RT }^{\mathbf{a}} \\
(\mathbf{m i n})\end{array}$ & $\begin{array}{c}\text { Precursor } \\
\text { ion }(\boldsymbol{m} / \boldsymbol{z})\end{array}$ & $\begin{array}{c}\text { Product } \\
\text { ion }(\boldsymbol{m} / \boldsymbol{z})\end{array}$ & $\begin{array}{c}\text { Collision } \\
\text { energy }(\mathbf{e V})\end{array}$ & $\begin{array}{c}\text { Tube lens } \\
(\mathbf{V})\end{array}$ \\
\hline Hydroxytyrosol & 1.7 & 153 & 123 & 17 & 54 \\
Syringealdehyde (IS) & 2.8 & 181 & 151 & 22 & 68 \\
Oleacein & 3.7 & 319 & 69 & 39 & 62 \\
Oleomissional & 3.9 & 377 & 275 & 16 & 103 \\
Oleocanthal & 4.5 & 303 & 59 & 17 & 56 \\
Oleokoronal & 4.6 & 361 & 291 & 17 & 88 \\
Oleocanthalic acid & 4.7 & 319 & 200 & 17 & 103 \\
Oleuropein aglycone & 5.1 & 377 & 275 & 16 & 103 \\
Ligstroside aglycone & 5.1 & 361 & 291 & 17 & 88 \\
Luteolin & 5.5 & 285 & 133 & 35 & 103 \\
Apigenin & 6 & 269 & 117 & 35 & 70 \\
\hline
\end{tabular}

${ }^{a}$ Retention time 
Table S2. Calibration models prepared for quantitative analysis of target phenols.

\begin{tabular}{lccc}
\hline \multicolumn{1}{c}{ Compound } & Calibration model & $\boldsymbol{R}^{\mathbf{2 a}}$ & $\begin{array}{c}\text { Calibration range } \\
(\mathbf{m g} / \mathbf{k g})\end{array}$ \\
\hline Hydroxytyrosol & $\mathrm{y}=(66019 \pm 1276) \mathrm{x}+16386 \pm 13139$ & 0.9970 & $1-20$ \\
Oleacein & $\mathrm{y}=(1762 \pm 36) \mathrm{x}-1802 \pm 368$ & 0.9967 & $1-20$ \\
Oleocanthal & $\mathrm{y}=(2375 \pm 83) \mathrm{x}+945 \pm 850$ & 0.9904 & $1-20$ \\
Oleocanthalic acid & $\mathrm{y}=(992116 \pm 2062) \mathrm{x}+54197 \pm 21226$ & 0.9982 & $1-20$ \\
Oleuropein aglycone & $\mathrm{y}=(101303 \pm 1926) \mathrm{x}+25928 \pm 49832$ & 0.9971 & $1-20$ \\
Ligstroside aglycone & $\mathrm{y}=(11064 \pm 297) \mathrm{x}+3914 \pm 3060$ & 0.9943 & $1-20$ \\
Luteolin & $\mathrm{y}=(139025 \pm 6430) \mathrm{x}+266806 \pm 66407$ & 0.9852 & $1-20$ \\
Apigenin & $\mathrm{y}=(89380 \pm 3414) \mathrm{x}+135534 \pm 35257$ & 0.9899 & $1-20$ \\
\hline
\end{tabular}

${ }^{\text {a Determination coefficient }}$

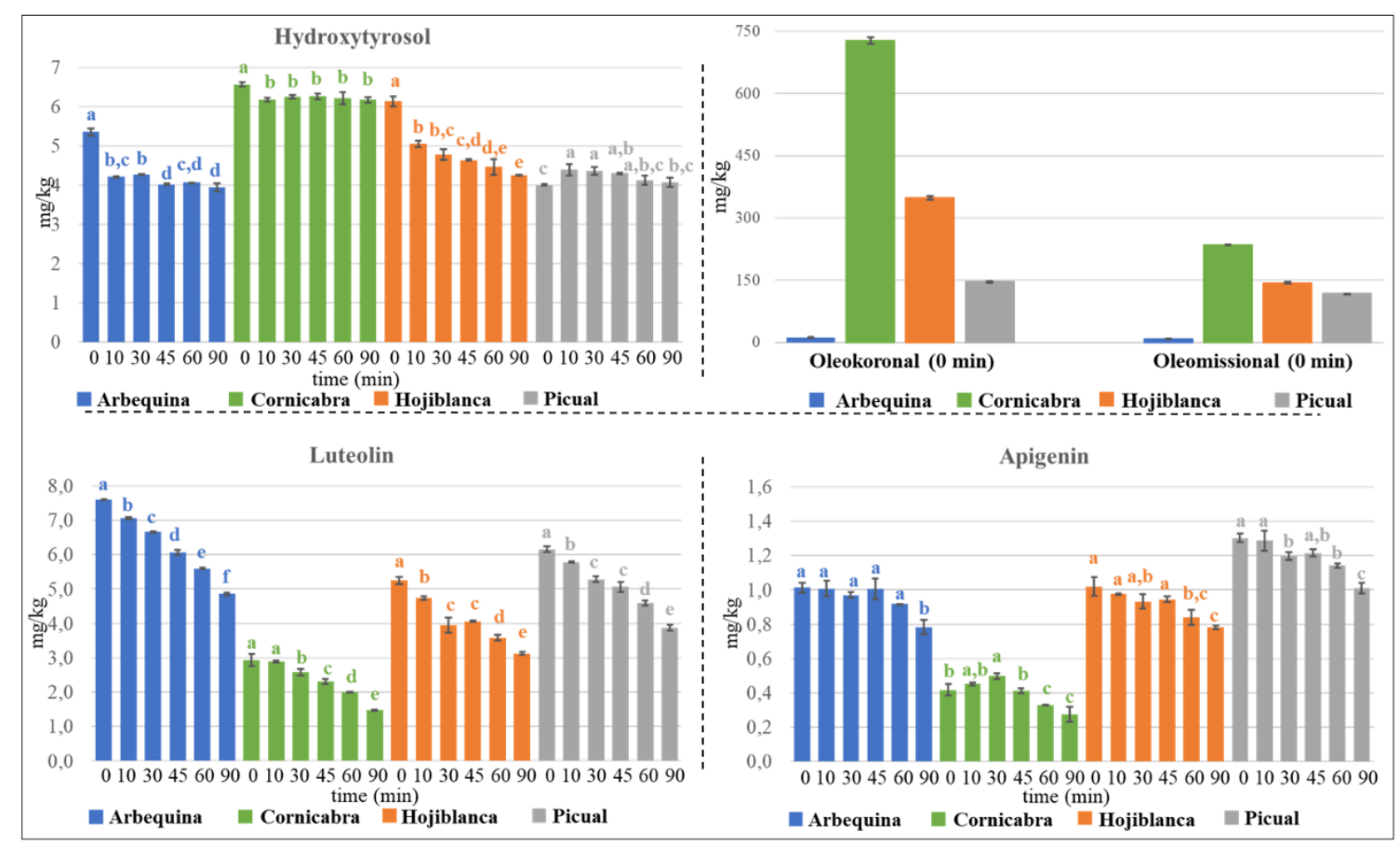

Figure S1. Variation in the concentration of other phenols in the four monocultivar EVOOs subjected to frying by analysis of aliquots sampled at $0,10,30,45,60$, and 90 min. 

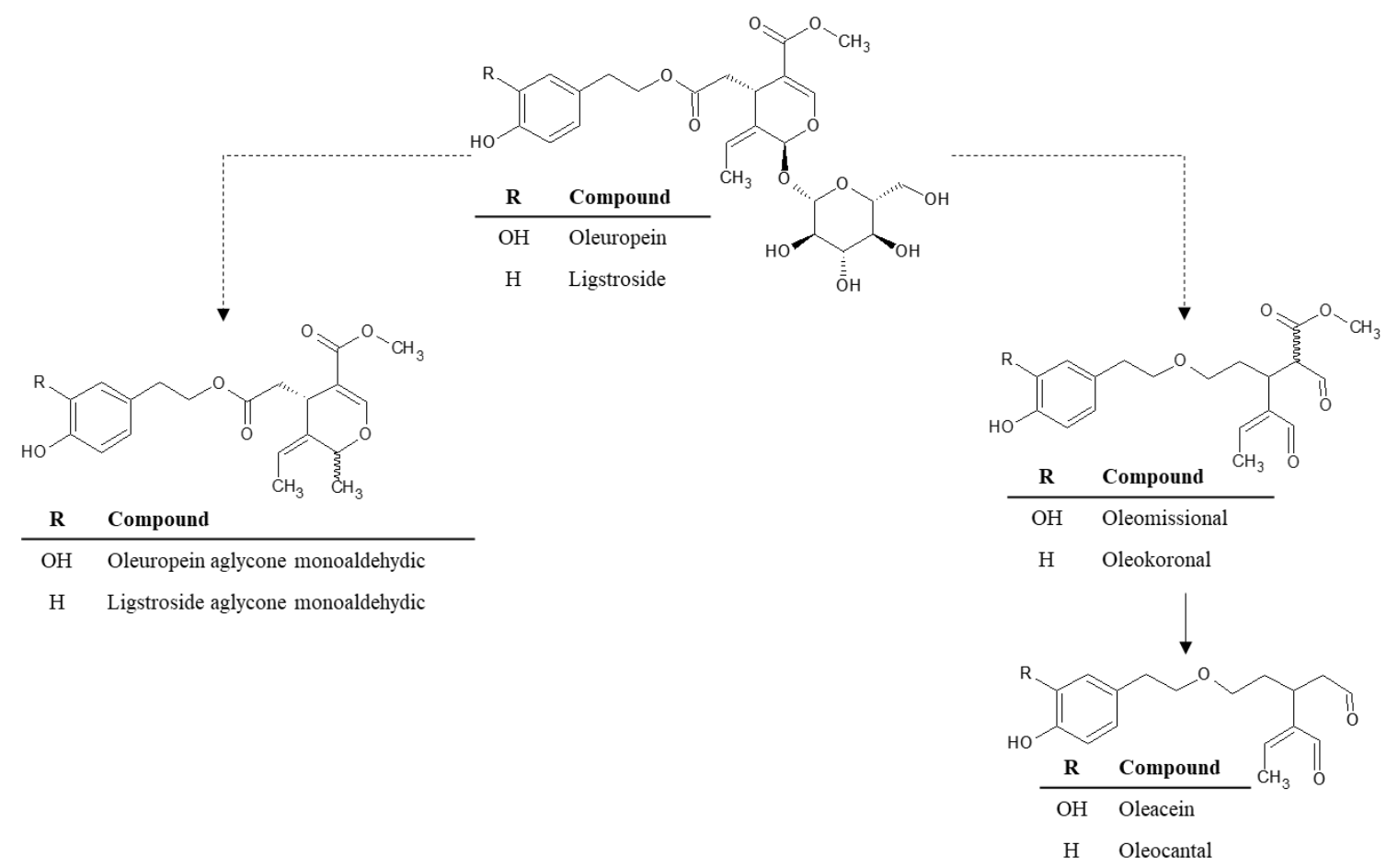

Figure S2. Mechanism proposed for the formation of main secoiridoid derivatives in olive oil (Diamantakos et al., 2020). The main alteration detected in the initial frying cycles $(10 \mathrm{~min})$ was the quantitative conversion of oleomissional/oleokoronal to oleacein/oleocanthal. 